This item was submitted to Loughborough's Research Repository by the author.

Items in Figshare are protected by copyright, with all rights reserved, unless otherwise indicated.

\title{
Tribology of rough ultra-film contacts in drug delivery devices
}

PLEASE CITE THE PUBLISHED VERSION

PUBLISHER

Professional Engineering Publishing / @ IMECHE

VERSION

VoR (Version of Record)

LICENCE

CC BY-NC-ND 4.0

REPOSITORY RECORD

Grimble, David W., Stephanos Theodossiades, Homer Rahnejat, and M. Wilby. 2019. "Tribology of Rough Ultra-film Contacts in Drug Delivery Devices”. figshare. https://hdl.handle.net/2134/4699. 
This item was submitted to Loughborough's Institutional Repository (https://dspace.lboro.ac.uk/) by the author and is made available under the following Creative Commons Licence conditions.

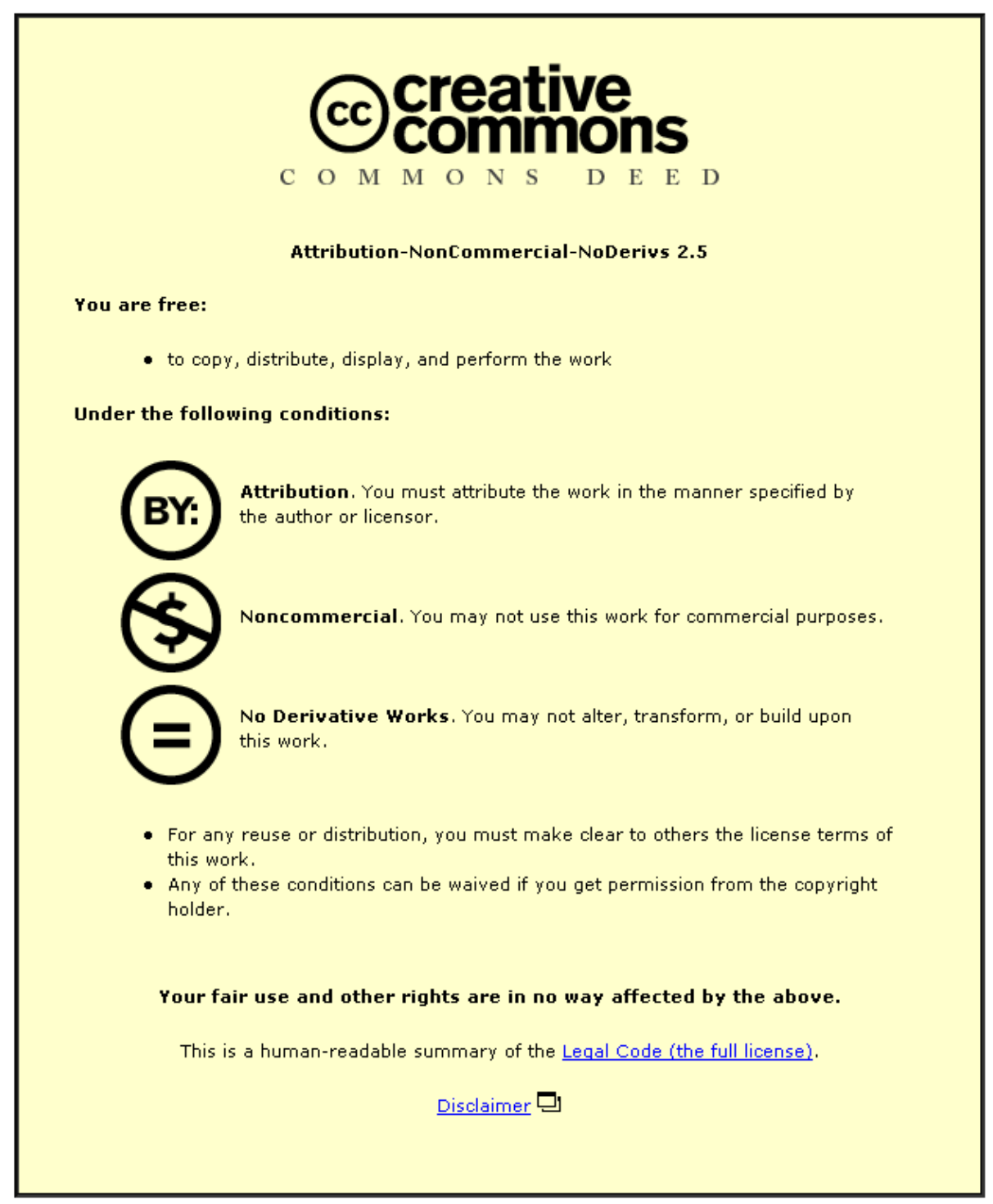

For the full text of this licence, please go to: http://creativecommons.org/licenses/by-nc-nd/2.5/ 


\title{
Tribology of rough ultra-film contacts in drug delivery devices
}

\author{
D W Grimble ${ }^{1}$, S Theodossiades $^{1 *}$, H Rahnejat $^{1}$, and M Wilby ${ }^{2}$ \\ ${ }^{1}$ Wolfson School of Mechanical and Manufacturing Engineering, University of Loughborough, Loughborough, UK \\ 23M Drug Delivery Systems, 3M Health, Loughborough, UK
}

The manuscript was received on 11 February 2008 and was accepted after revision for publication on 9 May 2008.

DOI: 10.1243/09544062JMES1047

\begin{abstract}
Elastomeric seals are extensively used in an assortment of drug delivery devices, such as syringes and pressurized metered dose inhalers. Although tribology of rubber seals and o-rings is reasonably well understood in engineering applications, the drug mixtures and formulations do not enjoy the required rheology to ensure coherent hydrodynamic action. In fact formation of uninterrupted hydrodynamic films is not actually sought in drug delivery devices, which often contain mixtures that are volatile when exposed to the environment. Furthermore, while engineering devices are often driven to overcome friction, many drug delivery systems are actuated manually and frequently by frail individuals. Therefore, the tribological problem is quite complex with many biological and environmental constraints. This paper highlights a parametric friction model for combined adhesive friction due to asperity interactions and non-Newtonian viscous action of the formulation. The model predicts the hysteretic behaviour of elastomeric seal contacts and conforms reasonably well to the experimental measurements of the same through actuation and release of inhaler valves.
\end{abstract}

Keywords: pressurized metered dose inhalers, rough thin-film contact, asperity interaction, adhesion

\section{INTRODUCTION}

Pressurized metered dose inhalers (pMDIs) have, in recent years, undergone several major changes, among them the replacement of chlorofluorocarbon by hydrofluoroalkane (HFA)-based propellants, chiefly due to the undesirable environmental effect of the former. One repercussion of this substitution has been the noted adverse effect on the tribological operational performance of the pMDIs, thought to be due to the poorer interaction of the new drug mixture containing HFAs in the rubber seal-to-stem conjunction of a typical pMDI [1]. With HFA only certain types of rubber material may be used as the porosity of the structure can lead to fluid digestion

\footnotetext{
*Corresponding author: Wolfson School of Mechanical and Manufacturing Engineering, University of Loughborough, Loughborough, Leicestershire LE11 3TU, UK. email: s.theodossiades@ lboro.ac.uk
}

and swelling, further exacerbating contact conditions. Thus, changes are required to the component materials in order to ensure compatibility with the new formulation and ensure smooth running of the devices. However, these have been largely of an empirical nature. The primary function of the rubber seal is to contain the volatile mixture under the requisite pressure within the canister. However, the inhalers' tribological performance is also very important.

There is a dearth of fundamental study of this tribological conjunction. Investigation of rubber-type seals have been mostly confined to o-rings or various seals operating mostly in the hydrodynamic regime of lubrication, for example using hydraulic fluids $[2,3]$. An initial analysis may be based on such approaches, but there are some significant differences; one of scale and the other of a complex fluid mixture whose behaviour deviates significantly from usual lubricants. The fluid in the conjunction, consisting mainly of HFA, has a very low dynamic viscosity of $0.21 \mathrm{mPa}$ at canister pressures of around $0.55 \mathrm{MPa}$ (gauge) and temperature of $20^{\circ} \mathrm{C}$. Thus, significant 
viscous action at low seal sliding speeds of $10 \mathrm{~mm} / \mathrm{min}$ is quite unlikely. This represents a typical actuation test speed and is used for comparative purposes in the analysis reported here. In normal use, however, it is recognized that speeds of $10000 \mathrm{~mm} / \mathrm{min}$ are commonplace. Consequently, under test conditions one would expect vanishingly small nano-conjunction between the seal and the housing. Given that the surface finish of both the seal and the housing are in excess of nanoscopic range, at best a mixed regime of lubrication would be expected. Thin surface films may exist in an adhered form to the asperity tips of the rubber seal, for which models proposed originally by Greenwood and Tripp [4] may be considered. Since the validity of the contact model for such narrow conjunctions is difficult to directly ascertain, the actuation effort for a pMDI can be measured in valve compression and release tests and compared with an equivalent model. This paper describes the tribological model developed and validates it against the aforementioned measurements.

\section{PRINCIPLE OF PMDI OPERATION}

Figure 1 shows a novel design of an inhaler valve that is to be modelled. It is a novel design as it does not employ a return spring, instead using the canister pressure. Furthermore, the seals are attached to the valve stem, rather than to the housing as in traditional valves. It has a canister which holds the drug mixture, which is referred to as the formulation. The formulation comprises an active substance (the drug), a propellant (HFA), and a surfactant (ethanol). There is also a metering valve, as well as an actuator (or adaptor). In tribological terms the surfactant clearly reduces the surface tension of the formulation, which otherwise would promote meniscus action, further contributing to the sticking of the contiguous surfaces.

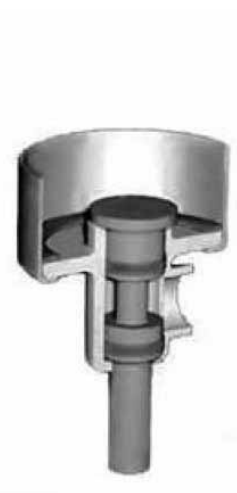

Start/Finish Position A

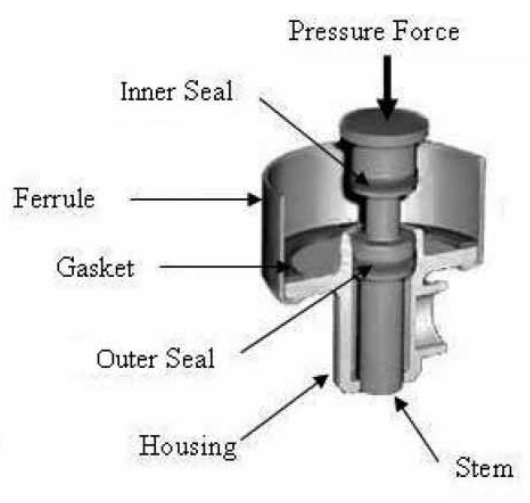

Midway Position D/E
Fig. 1 Schematic representation of an inhaler valve
The number of moving parts is kept to a minimum in order to reduce the effect of friction. The traditional spring loaded mechanism [1] is also replaced by the principle of a pressure-operated valve for the release stroke, thus reducing stick-slip effect due to spring actuated motions.

The canister pressure must be able to overcome the contact friction force of the seals, which can reduce the ease of actuation and potentially prohibit the return motion of the stem altogether. At the same time, good sealing is required to minimize leakage of the formulation. An acceptable leakage rate must be achieved and the design must be optimized to meet this requirement throughout the life of the device. This is especially difficult, when considering a good seal must be maintained over the course of some 200 actuations, typically over a period of 18-24 months.

\section{MODELLING OF RUBBER SEAL-HOUSING CONJUNCTION}

\subsection{Viscous effect}

The approaches undertaken for tribology of seals by Nikas [3] and Karaszkiewicz [5] can form the starting point in the current analysis with the main aim of ascertaining the likelihood of any hydrodynamic film. An empirical formula predicting film thickness has been presented in references [2] and [6] specifically for o-ring seals. The film thickness is given by

$$
h=4.4(\eta u)^{0.65} R^{0.56} W_{\mathrm{h}}^{-0.21} E^{\prime-0.44}
$$

where $E^{\prime}$ is the reduced elastic modulus of the rubber seal and housing contact as: $E^{\prime}=2\left(\left(1-v_{\mathrm{s}}^{2} / E_{\mathrm{s}}\right)+\right.$ $\left.\left(1-v_{\mathrm{f}}^{2} / E_{\mathrm{f}}\right)\right)^{-1} \cdot \eta=\eta_{0} e^{\alpha p}$ is the dynamic viscosity of the fluid, $u$ is the speed of entraining motion in the contact, being half the sliding speed of the seal relative to the stationary housing $(0.17 \mathrm{~m} / \mathrm{s})$ and $R$ is the equivalent radius of the partially conforming contact of seal to housing. $W_{\mathrm{h}}$ is the load per unit perimeter of the contact carried by a lubricant film. If the conjunction enjoys a coherent fluid film, then this load would equate that for a pressure loaded deformed seal when fitted in situ and subjected to a pressure, $p$ [2]

$$
W=w\left[\left(\frac{\pi}{6}\right)(2 \varepsilon+0.13) E_{\mathrm{s}}+\frac{v_{\mathrm{s}}}{1-v_{\mathrm{s}}} p\right]
$$

where $w$ is the effective width of the contact that the seal makes with the housing, when fitted in situ and subjected to a pressure, $p$. Thus, $W_{\mathrm{h}}=W$. The pressure in the canister containing the formulation is $p[\mathbf{5}]$, 

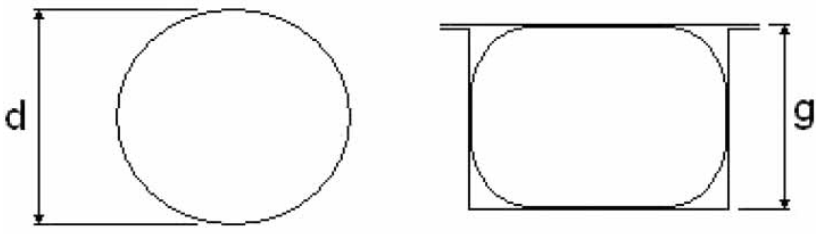

Fig. 2 Representation of a deformed pressurized seal

giving an effective contact width of

$$
\begin{aligned}
w= & \left\{(2 \varepsilon+0.13)+\left[0.39(1-\varepsilon)^{-1}-0.5(2 \varepsilon+0.13)\right]\right. \\
& \left.\times\left[1-\exp \left(-\frac{4.6 p}{E_{\mathrm{s}}}\right)\right]\right\} d
\end{aligned}
$$

$\varepsilon$ is the squeeze ratio for the deformed fitted and pressurized rubber seal, defined as: $\varepsilon=(d-g) / d$ (Fig. 2), with the value of 0.155 in the case studied here for a pressure of $0.55 \mathrm{MPa}$. However, the canister pressure alters in compression or release due to changes in chamber volume. In general, this is a complex issue. With the drug mixture, if the change of state is sufficiently slow for the vapour to be in equilibrium with its surrounding (i.e. to condense during compression), then one can assume that the pressure remains constant and equal to the vapour pressure. If the change of state is fast, one would normally assume isentropic compression. For this condition the pressure change due to volume can be considered as

$$
p_{\text {new }}=p_{\text {old }}\left(\frac{V_{\text {new }}}{V_{\text {old }}}\right)^{-1 / \gamma}
$$

where $\gamma=c_{\mathrm{p}} / c_{\mathrm{v}} \approx 1.2$ for HFA at $25^{\circ} \mathrm{C}$.

This change can then be followed by condensation and return to equilibrium. However, it is difficult to define 'fast' or 'slow' in this context. Therefore, when this effect is included in the model it will only return any significant changes under appropriate conditions.

Now using the physical and geometrical data provided in Table 1, the film thickness in the conjunction is obtained as $0.3 \mathrm{~nm}$, which is far less than the composite surface roughness of the counterfaces in contact. The results, therefore, suggest that a coherent fluid film cannot form in the conjunction, but it does not preclude molecules of the mixture acting as adsorbates on the asperity tips of the rough surfaces. Therefore, it is reasonable to assume, for the purpose of an initial analysis that the main mechanism for contact friction is due to combined boundary and viscous interactions. The boundary friction is due to asperity interactions and viscous contribution would correspond to shearing of the adsorbed surface fluid film at the asperity tips, behaving in a non-Newtonian manner.
Table 1 Physical parameters

\begin{tabular}{ll}
\hline Parameter & Value \\
\hline$D$ & $1.61 \mathrm{~mm}$ \\
$D$ & $6 \mathrm{~mm}$ \\
$E_{\mathrm{f}}$ & $2.6 \mathrm{GPa}$ \\
$E_{\mathrm{s}}$ & $2.5 \mathrm{MPa}$ \\
$G$ & $1.36 \mathrm{~mm}$ \\
$p$ & $0.55 \mathrm{MPa}$ \\
$R$ & $0.805 \mathrm{~mm}$ \\
$u$ & $10 \mathrm{~mm} / \mathrm{min}^{-9} \mathrm{~Pa}^{-1}$ \\
$\alpha$ & $2.8 \times 10^{-1} \times 10^{-3} \mathrm{~Pa} \mathrm{~s}$ \\
$\eta_{0}$ & $0.211 \times$ \\
$\Sigma \beta \sigma$ & 0.1289 \\
$\tau_{0}$ & $3.5 \mathrm{MPa}$ \\
$v_{\mathrm{f}}$ & 0.35 \\
$v_{\mathrm{s}}$ & 0.49 \\
\hline
\end{tabular}

\subsection{Effect of asperity interactions}

A friction model based on asperity interaction with some minor contribution due to viscous shear of wet asperity tips is developed, where: $F=F_{\mathrm{b}}+F_{\mathrm{v}}$. The boundary contribution is obtained using the method proposed by Greenwood and Tripp [4]. A Gaussian distribution of asperity heights is assumed and an equivalent area of asperities making direct contact is calculated as

$$
A_{\mathrm{a}}=\pi^{2} A(\varsigma \beta \sigma)^{2} F_{2}(\lambda)
$$

where $A$ is the apparent area of contact, $\varsigma$ the surface density of asperity peaks, $\beta$ the average radius of curvature of asperity tips, and $\sigma$ the root mean square of counterfaces' surface roughness. $\lambda=h / \sigma$ is the film or separation parameter.

The load supported by the asperities in direct contact can then be calculated using [4]

$$
W_{\mathrm{a}}=\frac{8 \sqrt{2}}{15} A \pi(\varsigma \beta \sigma)^{2} \sqrt{\frac{\sigma}{\beta}} E^{\prime} F_{5 / 2}(\lambda)
$$

The statistical functions $F_{2}(\lambda)$ and $F_{5 / 2}(\lambda)$ are evaluated from the integral

$$
F_{n}(\lambda)=\frac{1}{\sqrt{2 \pi}} \int_{\lambda}^{\infty}(s-\lambda)^{n} e^{-s^{2} / 2} \mathrm{~d} s
$$

However, it is more convenient to use a polynomial representation in order to calculate these terms as shown in Fig. 3. Note that for a value of $\lambda=0.0015$ (in the case reported here), the values obtained nearly represent the intercept of the curves with the ordinate in the figure.

In order to use the proposed asperity interaction model, it is necessary to use the correct parameters; $\beta, \sigma, \varsigma$. The individual parameters are difficult to obtain. However, values for the product of the three terms have been suggested, for example in the range $0.042-0.07$ for the metal surfaces by Grosch [7] and in 


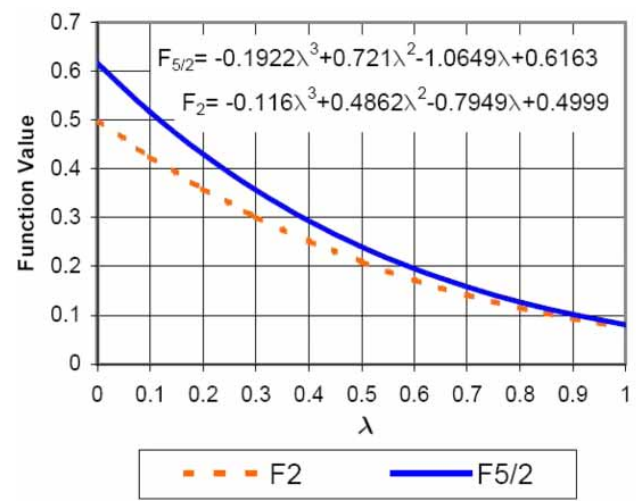

Fig. 3 Statistical approximation of surfaces (after Greenwood and Tripp [4])

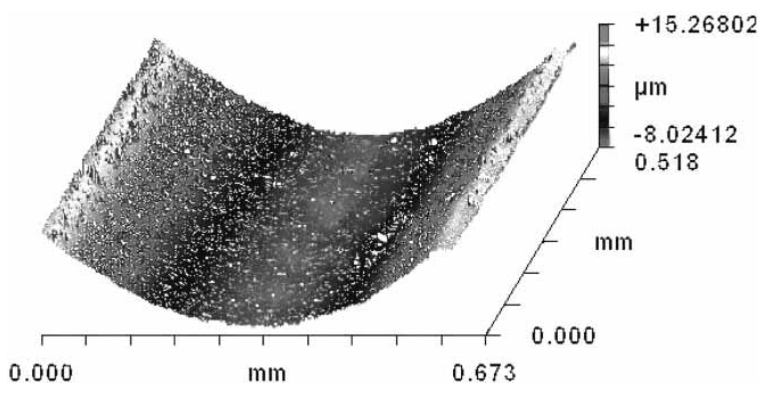

Fig. 4 Example surface profile scan

the range 0.09-0.15 for polymer-elastomeric contacts (such as that of a rubber seal against a polymeric housing) by Fuller and Tabor [8]. In order to ensure that the correct values were used for the model, component samples were obtained which were then examined using an interferometric scanning machine (Fig. 4). A value of 0.12 was obtained for the product of these parameters which is in the range of those suggested by Fuller and Tabor $[\mathbf{8}]$.

\subsection{Combined viscous and boundary considerations}

It is necessary to combine the effects of asperity interactions and viscous contribution in carrying the contact load generated by applied canister pressure and seal deformation, as well as predicting friction. The following iterative procedure is used.

Step 1. For a given contact load the lubricant film thickness is calculated from equation (1).

Step 2. For root mean square surface roughness of contiguous surfaces and product of parameters $\beta, \gamma$, and $\varsigma$, using equation (5), the asperity contact area is determined.

Step 3. The proportion of load carried by the asperities, $W_{\mathrm{a}}$, is determined using equation (6).

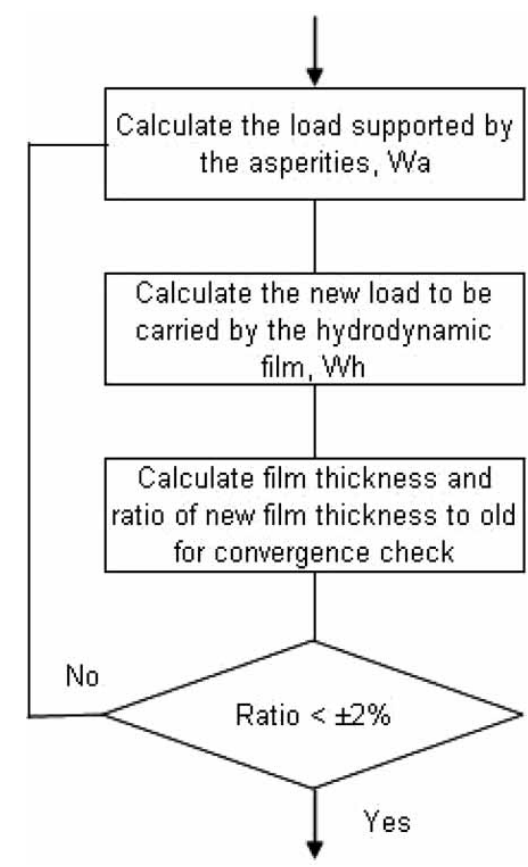

Fig. 5 Basic approach for convergence of asperity/fluid load share

Step 4. The share of load carried by the adsorbed fluid on asperity tips is determined as: $W_{\mathrm{h}}=W-W_{\mathrm{a}}$. This reduced load has the effect of increasing the film of fluid.

Step 5. The area of asperity contact is adjusted and, thus, the new load carried by the asperities is obtained.

Step 6. The change in the proportion of load carried by asperities within successive iteration steps is checked as to ascertain that its value is within a specified limit (Fig. 5). If the condition is met, then the overall friction is calculated. Otherwise, the iteration process continues.

Step 7. Upon convergence, adhesive friction due to asperity-tip interactions is calculated as

$$
F_{\mathrm{b}}=\tau_{0} A_{\mathrm{a}}+m W_{\mathrm{a}}
$$

where $\tau_{0}$ is the Eyring shear stress of the lubricant, with the limiting value of $3.5 \mathrm{MPa}$, and $m$ is the pressure coefficient of boundary shear strength, with a value of 0.13 [9]. The viscous friction for such ultra-thin films is non-Newtonian and obtained as

$$
F_{\mathrm{v}}=\tau\left(A-A_{\mathrm{a}}\right)
$$

where $\tau=u \eta / h$ when $\tau<\tau_{0}$, otherwise: $\tau=\tau_{0}+\gamma p$, where

$$
p=\frac{W-W_{\mathrm{a}}}{A_{\mathrm{a}}}
$$




\section{MEASUREMENTS AND MODEL VALIDATION}

Model predictions were compared with a series of compression tests carried out for the pMDIs at a constant rate of $20 \mathrm{~mm} / \mathrm{min}$ (a sliding velocity of $0.34 \mathrm{~m} / \mathrm{s}$ ). The valves were then released to return under the action of canister pressure. Figure 6 shows the typical characteristics obtained experimentally shown in full line. This is the hysteretic behaviour of the device; its force-extension/deflection characteristics. Compression force is applied at point A (Fig. 1) and follows the steep curve AB. The force along this part of the characteristic plot is the static friction due to both seals. Motion commences at point $\mathrm{B}$, where the static friction peak at the onset of motion is visible. Thereon, steady uniform motion dictates no inertial effect.

From point B, the characteristics follow the pressure gradients across the two seals (shown in Fig. 1) and the kinetic friction during the compression phase. As the actuation continues, the inner-most seal leaves the contact with the housing bore at point $\mathrm{C}$, resulting in a sharp drop in friction. By point $\mathrm{D}$, the valve cannot be compressed further and the compression testing machine reaches the housing, causing a sharp increase in the measured force. At this point the transition to valve release begins. From point D (Fig. 1) the return force is the net difference between the pressure induced force and the friction of the outer seal's contact. In the region $\mathrm{BD}$, the resultant force shown in Fig. 6 is that necessary to overcome the opposing forces of the combined seals' friction force and that of the canister pressure. As point $\mathrm{E}$ is reached, and in the region $E A$, the resisting force attempts to prevent valve return at a rate faster than that specified. This force is due to the canister pressure.
At point $\mathrm{E}$ the valve stem begins to move again and the pressure return force, discounting friction due to one seal is recorded. Point $\mathrm{F}$ indicates an increase in system friction as the inner-most seal re-enters the housing, resulting in a reduction in the return force of the overall device as seen in Fig. 6 . Finally, the characteristics return to point $\mathrm{A}$ as the valve is entirely released and comes to rest.

The dotted line in Fig. 6 represents the predicted characteristics using the described friction model applied at both seal-to-housing contacts in a dynamic model, where the stem is moved at the sliding speed of $20 \mathrm{~mm} / \mathrm{min}$. As can be observed, reasonable agreement is found with the measured results. The predicted film thickness from equation (1) for both seals is in the range $2-4 \mathrm{~nm}$, which indicates that contribution due to viscous friction is very small compared with the boundary contribution. The variation in the outer seal film thickness is shown in Fig. 7. As can be observed throughout the compression-release cycle the film thickness is very small indeed, indicating that contribution due to viscous friction is almost negligible. This is verified by the inner and outer seal predicted friction in Figs 8 and 9 (the inset to the figures show the viscous contribution). Note that boundary friction is dominant, accounting for more than 95 per cent of the total friction. In all the figures the lettering A-F corresponds to the same instances as already described for Fig. 6 . The static friction is represented by the ordinate value corresponding to $\mathrm{AB}$. This indicates an inner seal friction of approximately $5 \mathrm{~N}$ and a corresponding value of $8 \mathrm{~N}$ for the outer seal, which represent the effort $A B$ on Fig. 6. This static friction is entirely contributed by boundary interactions, given by equation (8) (note that there would be

forces@20 mm/min

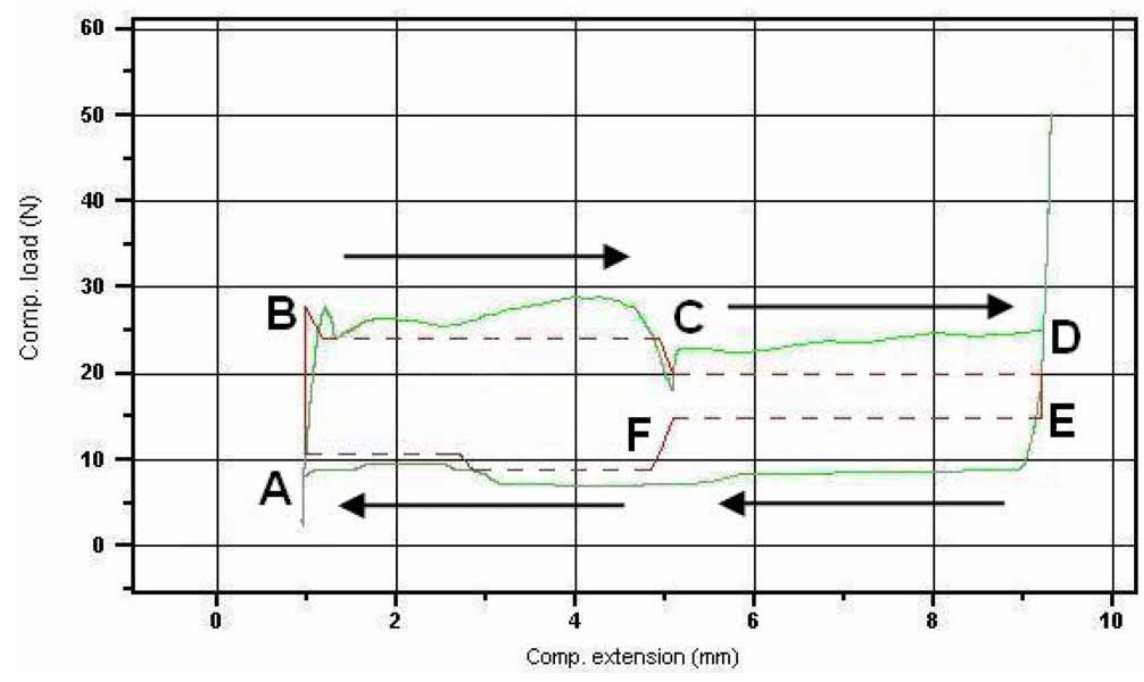

Fig. 6 Measured and predicted valve actuation characteristics 


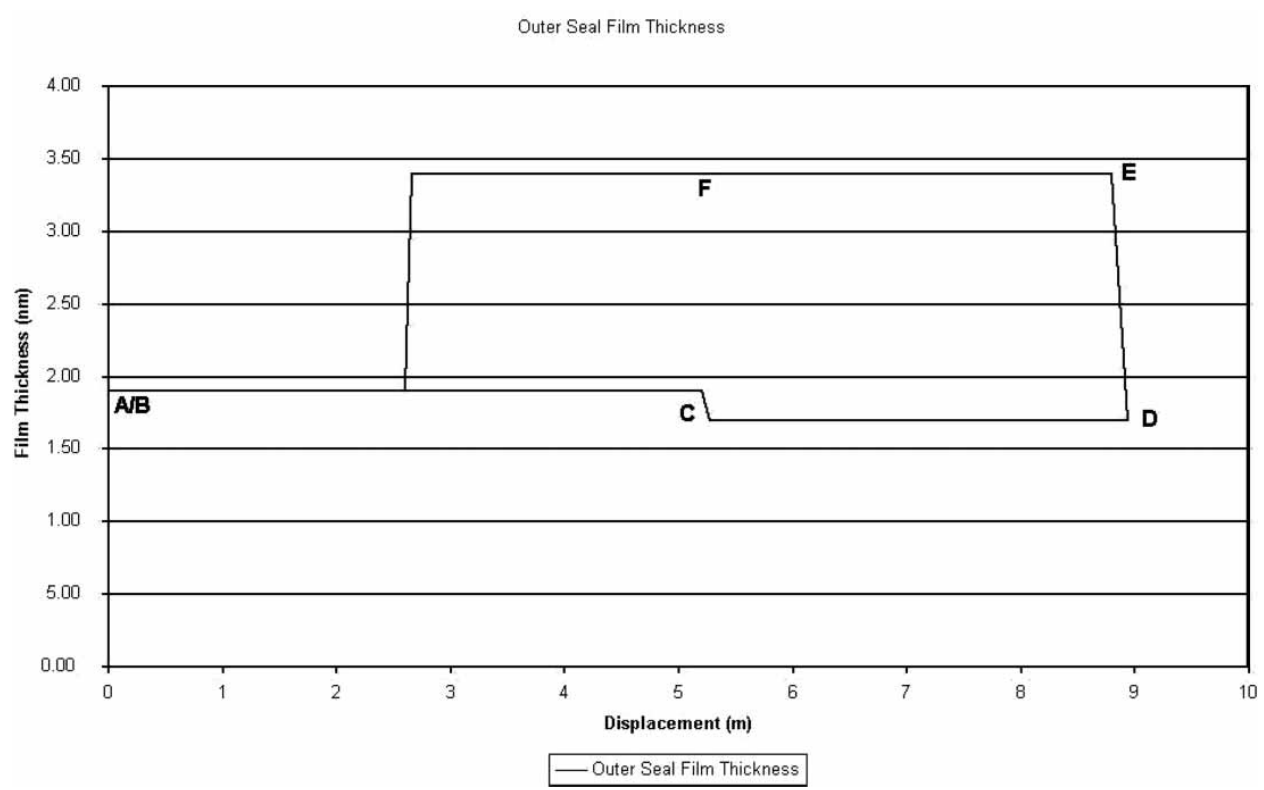

Fig. 7 Cyclic variation of outer seal-to-housing film thickness

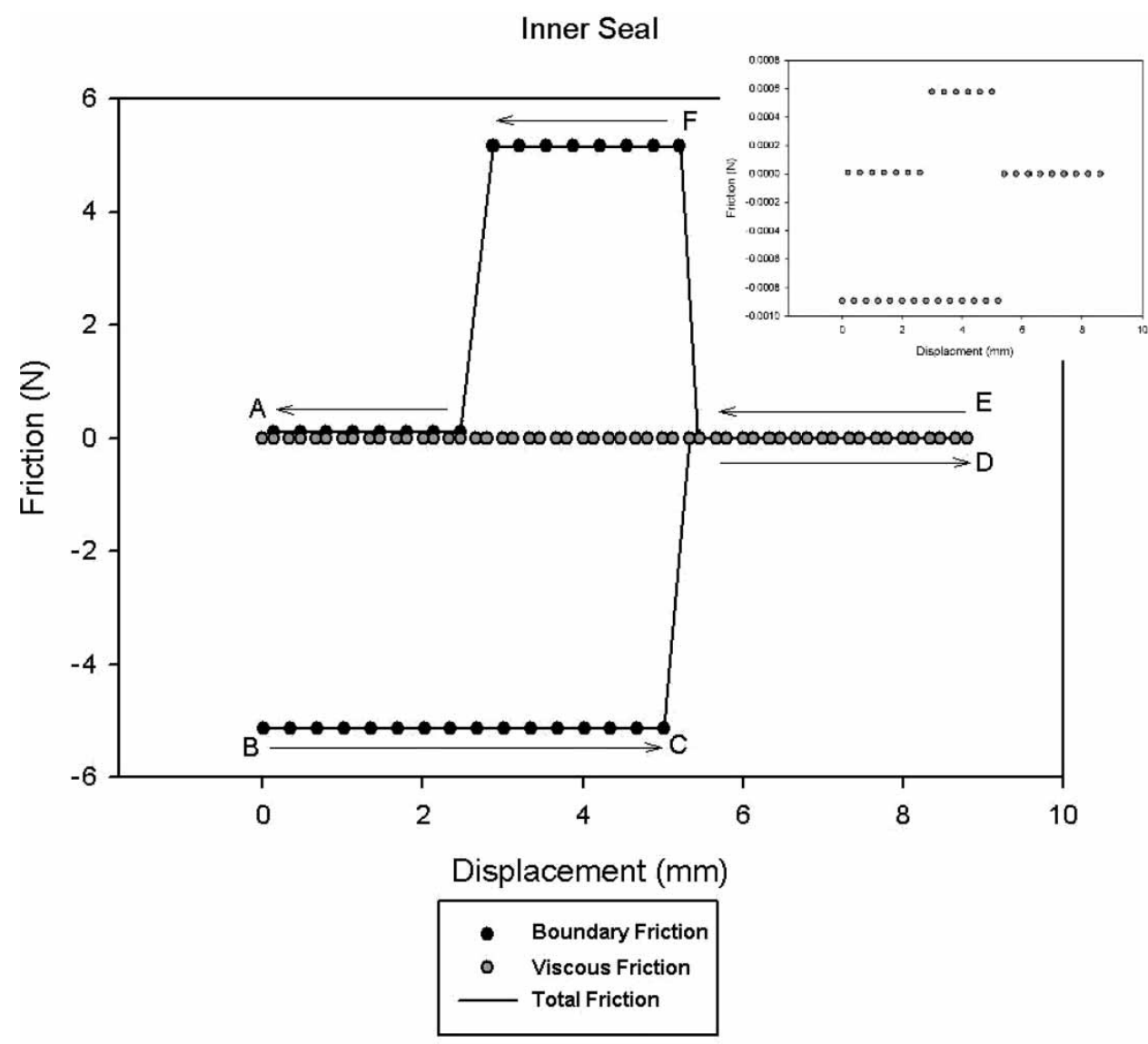

Fig. 8 Inner seal friction

no contribution due to any viscous action with cessation of entraining motion). A seals' friction remains almost unaltered for valve motion corresponding to $\mathrm{BC}$, because of the uniform nature of the motion (Figs 8 and 9). At $C$ the inner seal leaves contact and friction contribution from it ceases, while an increased pressure differential acting across the outer seal results in a larger area of asperity contact (thus an increased value 


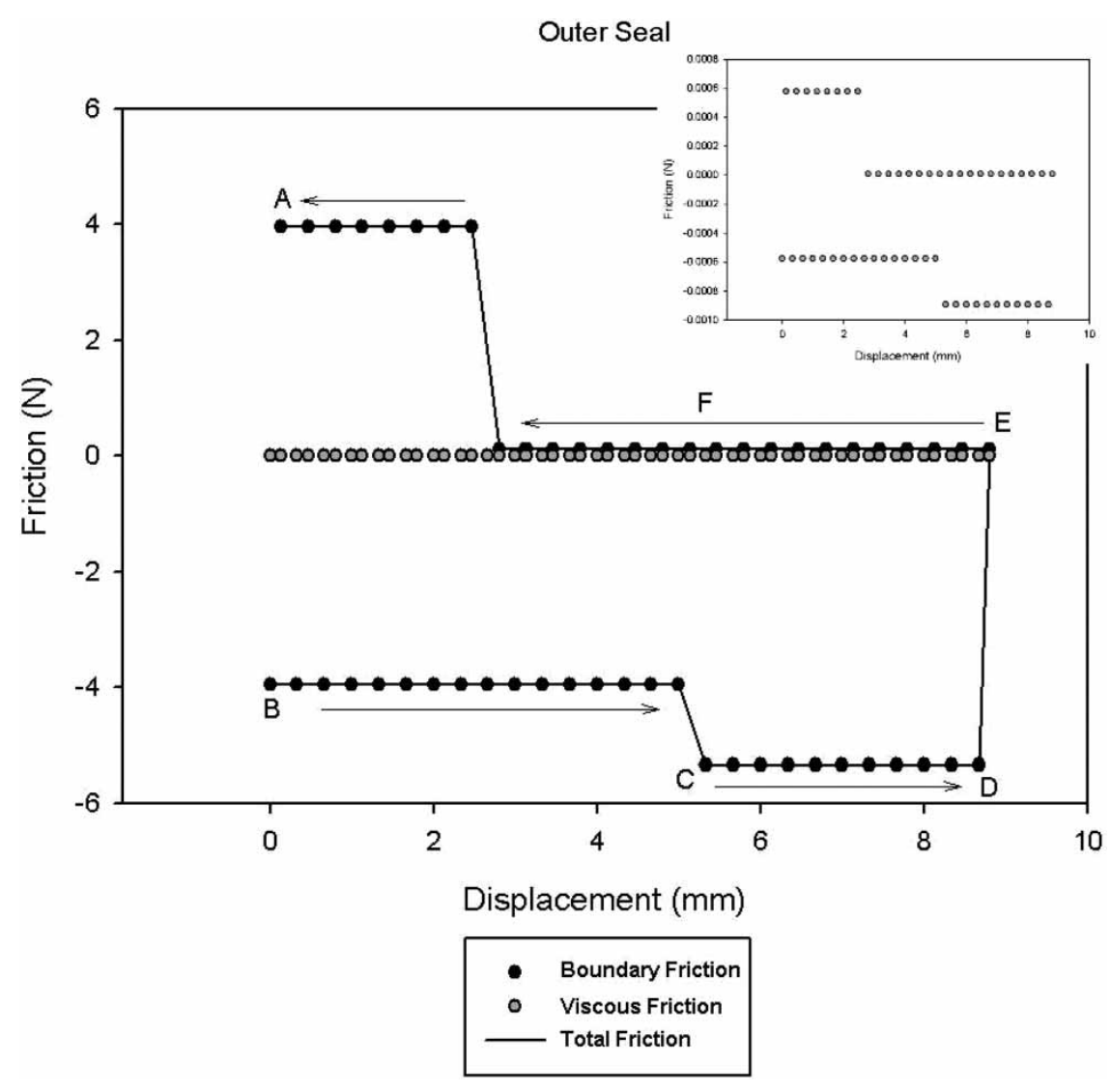

Fig. 9 Outer seal friction

of $W_{\mathrm{a}}$ and hence friction, equation (8)). The outer seal friction remains almost unaltered due to the uniform motion of the valve up to point $\mathrm{D}$ (end of compression stroke), whilst the inner seal is still out of contact. The return action begins immediately. At $\mathrm{F}$ the inner seal comes into contact with dramatically increased friction, therefore causing a reduction in the return force in Fig. 6. The pressure differential across the outer seal drives it until aerosol release in the interval FA, while the inner seal comes to rest.

The differences between the predicted results and the experimental measurements are because of a number of factors, unaccounted for in the model. First, the rubber seals digest a certain amount of formulation and swell, thus altering the contact area. Second, the boundary friction model employed here presumes dominance of adhesive friction between asperity tippairs, thus the effect of deformation (ploughing) friction in their potential oblique interactions is ignored. Finally, small menisci are expected to form between the asperity tips of the seals and those of the housing, which have been ignored in this initial analysis. Another important contributor to friction is van der Waals forces which can contribute significantly to static friction under dry contact condition. Under 'wet' conditions van der Waals interactions are considerably reduced. Point E in Fig. 6 seems to suggest this effect in transition from a momentarily drying contact to a wet case. These considerations form the basis for more in-depth future research.

\section{CONCLUSION}

The hysteretic characteristics of the pMDIs determine the required effort in valve actuation, which is an important performance measure due to the range of users from the very young to the very elderly. This requirement together with the need for proper sealing of volatile formulations, assurance of precise dosage delivery and robustness for many actuations govern the design of pMDI devices. Unfortunately, these prerequisites result in conjunctions that are tribologically poor due to environmental and bio-compatibility constraints imposed on the use of propellants with poor rheology in tribological terms. Thus, excessive friction can lead to a greater actuation effort and poor stem return repeatability (affected by canister pressure overcoming the conjunctional friction). This may result in unrepeatable and inaccurate successive actuations leading to poor drug delivery characteristics. The paper has shown the importance of developing a parametric friction model, which can be expanded to include other previously mentioned phenomena, thus 
acting as a predictive tool in the development of a new generation of pMDIs.

\section{ACKNOWLEDGEMENTS}

The authors would like to express their gratitude to Engineering and Physical Sciences Research Council (EPSRC) and 3M for their financial support extended to this research project.

\section{REFERENCES}

1 Purewal, T. S. and Grant, D. J. W. Metered dose inhaler technology, 1978 (Taylor \& Francis, London).

2 Karaszkiewicz, A. Hydrodynamic lubrication of rubber seals for reciprocating motion; leakage of seals with an O-ring. Tribol. Int., 1988, 21(6), 361-367.

3 Nikas, G. K. Transient elastohydrodynamic lubrication of rectangular elastomeric seals for linear hydraulic actuators. Proc. Instn Mech. Engrs, Part J: J. Engineering Tribology, 2003, 217, 461-473.

4 Greenwood, J. A. and Tripp, J. H. The contact of two nominally flat rough surfaces. Proc. Instn Mech. Engrs, 1970-1971, 185, 625-633.

5 Karaszkiewicz, A. Geometry and contact pressure of an o-ring mounted in a seal groove. Ind. Eng. Chem. Res. Dev., 1990, 29, 2134-2137.

6 Karaszkiewicz, A. Hydrodynamics of rubber seals for reciprocating motion, lubricating film thickness, and out-leakage of O-seals. Ind. Eng. Chem. Res. 1987, 26, 2180-2185.

7 Grosch, K. A. The relation between the friction and viscoelastic properties of rubber. Proc. R. Soc. A, 1963, 274, 21-39.

8 Fuller, K. N. G. and Tabor, D. The effect of surface roughness on the adhesion of elastic solids. Proc. R. Soc. Lond., 1975, 345(1642), 327-342.

9 Briscoe, B. J, Scruton, B., and Willis, F. R. The shear strength of thin lubricant films. Proc. R. Soc. Lond. A., 1973, 333, 99-114.

\section{APPENDIX}

\section{Notation}

A apparent area of contact

$A_{\mathrm{a}} \quad$ apparent area of asperity contact specific heat capacity at constant pressure specific heat capacity at constant volume undeformed cross-sectional seal diameter outer diameter of seal elastic modulus of the housing elastic modulus of the seal reduced elastic modulus statistical function of surface film/separation statistical function of surface film/separation total friction force boundary friction viscous friction seal groove height film thickness film thickness, where $\mathrm{d} p / \mathrm{d} x=0$ minimum film thickness the pressure coefficient of boundary shear strength working pressure working pressure at current canister volume working pressure at initial canister volume effective radius of contact speed of entraining motion current inhaler canister volume initial inhaler canister volume loaded contact width load supported by asperity contact load carried by hydrodynamic action per unit length of the seal total contact load per unit length of the seal

piezo-viscosity index average radius of curvature of asperity tips adiabatic index squeeze ratio dynamic viscosity film thickness ratio surface density of asperity peaks root mean square of counterfaces' surface roughness Eyring shear stress Poisson's ratio for the housing Poisson's ratio for the seal 